\title{
Evaluación de costos en la etapa de lixiviación en un proceso de blanqueamiento de arcilla caolinítica
}

\author{
A. D. Toache a,," L. E. Hernández ${ }^{\mathrm{a}}$, A. M. Bolarínª Félix Sánchez ${ }^{\mathrm{a}}$ \\ a Área Académica de Ciencias de la Tierra y Materiales, UAEH, Carr. Pachuca-Tulancingo Km 4.5, C.P. 42184, Mineral de la Reforma, Hgo., México.
}

\section{Resumen}

En este trabajo se hizo una evaluación de costos en la etapa de lixiviación en un proceso de blanqueamiento de arcillas caoliníticas, para lo cual se analizaron dos diferentes métodos de saturación de los licores de lixiviación. En ambos métodos se requiere de agitación constante y temperatura de $90^{\circ} \mathrm{C}$ y se utilizan como reactivos ácido cítrico y tiosulfato de sodio diluidos en agua desionizada para remover las impurezas presentes en la arcilla caolinítica. La diferencia es que en uno de los procedimientos se lixiviaron $5 \mathrm{~kg}$ de ésta, por períodos mayores a 240 minutos, lo que implica emplear mayor cantidad de energía en el reactor y en un segundo procedimiento se recircularon los licores de lixiviación para blanquear $10 \mathrm{~kg}$ de arcilla caolinítica, sin necesidad de añadir mayor cantidad de reactivos. Se investigaron los costos de los reactivos, del consumo de energía eléctrica del reactor, del consumo de agua en servicios industriales y el costo de la arcilla caolinítica extraído a pie de mina y de alta pureza. Comparando costos y analizando el posible precio de venta, se concluyó que el método más factible, para el blanqueamiento de dicha arcilla, es en el que se hace la recirculación de los licores de lixiviación para tratar nuevos lotes de arcilla.

Palabras Clave: Análisis, Costos, Caolín, Lixiviación, Planta industrial.

\section{Introducción}

En México existen importantes yacimientos de caolín ubicados en los estados de Chihuahua, Guanajuato, Michoacán, Veracruz e Hidalgo (Departamento de Estadística Minera, 2010); sin embargo, el mineral extraído de estos yacimientos contiene diferentes contaminantes e impurezas que minimizan su aplicación en diversas industrias.

Los métodos de remoción de dichos contaminantes son diversos e incluyen procesos tales como separación magnética, flotación por espuma, floculación selectiva y lixiviación, siendo esta última una de las más empleadas. Esta última, se basa en la remoción o reducción de los óxidos de hierro, debido a que este elemento es el principal contaminante de las arcillas caoliníticas, causante de su coloración café-amarillo (beige) (Cameselle, Nuñez, Lema \& Pais, 1995). Los óxidos de hierro se encuentra presente en pequeñas cantidades en forma de óxidos e hidróxidos, como la hematita, magnetita, goethita, lepidocrocita y ferrihidrita (Ambikandebi \& Lalithambika, 2000).

En este sentido, por la vía hidrometalúrgica (lixiviando) se han reportado diferentes métodos de remoción de hierro de la arcilla caolinítica, con distintos agentes y condiciones de trabajo, todos ellos con excelentes resultados que permiten lograr la calidad del caolín que las empresas requieren (Subsecretaria de Minería, 2017) (Medinaceli \& Trujillo 2016). Por ejemplo, una de estas investigaciones propone la utilización de ácido fosfórico $[3.0 \mathrm{M}]$, a temperatura de $100^{\circ} \mathrm{C}$ durante un período de 120 minutos, logrando obtener hasta un 98.65\% de disolución de hierro de la arcilla caolinítica (Hernández et al., 2015). En otro trabajo publicado por Legorreta et al. (2015), se utiliza una concentración de $0.5 \mathrm{M}$ ácido oxálico como agente lixiviante a temperatura de $100^{\circ} \mathrm{C}$ y $\mathrm{pH}$ de 1.5 durante 60 minutos, logrando extracciones de hierro de $96 \%$. A pesar de los buenos resultados reportados en los trabajos antes mencionados, Olvera et al. (2012 y 2014) realizaron, mediante lixiviación, la limpieza de este tipo de arcillas caoliníticas, pero utilizando ácido cítrico y tiosulfato de sodio a temperatura de $90^{\circ} \mathrm{C} \mathrm{y} \mathrm{pH} \mathrm{3,} \mathrm{eliminando} \mathrm{hasta} \mathrm{un}$ 99\% de Fe (principal contaminante) de la arcilla caolinítica. El utilizar este tipo de reactivos orgánicos permitió reducir $10^{\circ} \mathrm{C}$ la temperatura de trabajo, a diferencia de los trabajos reportados por Hernández et al. (2015), lo cual representa un gran ahorro si se quisiera implementar esto a un nivel mayor que el de laboratorio.

Al revisar la bibliografía, también pudo observarse que en ninguna de las investigaciones reportadas se ha efectuado un análisis económico, de cadena de valor o de costos, razón por la cual en el presente trabajo se realiza un análisis económico, partiendo de las condiciones propuesta por Olvera et al. (2014) de dos diferentes métodos utilizados durante el proceso de lixiviación, con la finalidad de establecer tanto los beneficios y ventajas económicas, como los costos para determinar el método más factible a seguir. 


\section{Metodología}

\subsection{Procesos de lixiviación utilizados en el blanqueo de la arcilla caolinítica considerados para la evaluación económica}

Dentro del proceso de blanqueo de la arcilla caolinítica por la vía hidrometalúrgica, se obtienen licores de lixiviación con diversos contaminantes, como el hierro (Fe) y el titanio (Ti) (Toache, 2015), cuyas concentraciones están por encima de los límites máximos permisibles establecidos en 2017 por la Organización Mundial de la Salud (OMS, 2017) y en México en la NOM-001-SEMARNAT y en la NOM-004SEMARNAT, lo que impide que estos efluentes puedan ser vertidos al medio ambiente, razón por la cual es importante tratarlos antes de desecharlos. Por tal motivo se experimentó con dos métodos de tratamiento los cuales consistieron en su saturación mediante dos variantes.

Un primer experimento se basó en tratar (alimentar) una doble cantidad de arcilla caolinítica a la utilizada normalmente en el método establecido previamente por Olvera et al. (2014), con la finalidad de determinar la posibilidad de saturar el licor de lixiviación en un período de tiempo igual (proceso 1). El siguiente experimento se basó en recircular (reutilizar) los licores de lixiviación, una y otra vez hasta que éstos se saturaran y ya no dieran los resultados esperados en el blanqueo de la arcilla caolinítica (proceso 2).

\subsection{Análisis de costos}

El estudio o análisis de costos permite determinar la rentabilidad futura de un producto o servicio que será introducido al mercado; además en la actualidad es importante orientarse estratégicamente a producir con el menor costo y la mayor calidad posible, permitiendo lograr una posición competitiva (Arredondo, 2015). Para alcanzar este objetivo es importante definir los tipos de costos asociados al proceso o procesos que se analizarán.

Un costo puede definirse como un sacrificio o utilización de recursos para un propósito en particular y, de acuerdo a lo que se quiere medir, éstos tienen diferentes clasificaciones (Jiménez \& Espinoza, 2007); para este estudio se utilizarán los costos directos e indirectos y los costos variables y fijos.

Los costos directos son aquellos que se derivan de la existencia de lo que se trata de determinar, por ejemplo, los materiales directos destinados a la fabricación de un producto. Los costos indirectos son aquellos con los que la identificación con un objeto de costos específicos es muy difícil, por ejemplo, costos de supervisión, de mantenimiento, entre otros. Un costo variable cambia en total en proporción a los cambios en el nivel relacionado del volumen o actividad total; por el contrario, un costo fijo permanece sin cambios en total por un período dado, pese a grandes cambios en el nivel relacionado con la actividad o volumen totales (Horngren, Data \& Foster, 2007).

\subsection{Cadena de Valor}

La cadena de valor es una técnica original de Michel Porter (1986) que tuvo como finalidad obtener una ventaja competitiva sobre los competidores clave. Este análisis permite identificar los distintos costos en que incurre una organización a través de las distintas actividades que conforman su proceso productivo, por lo que constituye un elemento indispensable para determinar la estructura de costos de una compañía (Quintero \& Sánchez, 2006), en este caso de la puesta en marcha de una planta piloto de purificación de arcillas caoliníticas.

Frances (2001) menciona que la cadena de valor está conformada por una serie de etapas de agregación de valía y de aplicación general en los procesos productivos, proporcionando un esquema coherente para diagnosticar la posición de una empresa y un procedimiento para definir acciones tendentes a desarrollar una ventaja competitiva sostenible.

\subsection{Procedimiento}

Para el análisis de costos se analizaron los recursos y materiales utilizados en los procesos de lixiviación de la arcilla caolinítica descritos de manera general en la sección 2.1. El estudio se realizó considerando que dichos procesos se efectúan en 240 minutos.

Un primer proceso (Figura 1) consistió en lixiviar $5 \mathrm{~kg}$ de arcilla caolinítica. Se eleva la temperatura del agua $(50 \mathrm{~L})$ hasta $90^{\circ} \mathrm{C}$, agitando y agregando ácido cítrico [0.9M], tiosulfato de sodio [0.5M]. Posteriormente, se ajusta el $\mathrm{pH}$ a 3 con hidróxido de sodio y, por último, se adicionan $5 \mathrm{~kg}$ de arcilla caolinítica. La agitación y la temperatura de $90^{\circ} \mathrm{C}$ se mantienen constantes durante un período de 240 minutos. Posteriormente se filtra la mezcla, separando el licor de lixiviación (que se trata antes de ser vertido al medio ambiente) y el caolín blanqueado.

En la Figura 2 se muestra el segundo proceso en el que se recircularon los licores provenientes de la lixiviación de la arcilla caolinítica de la forma siguiente: en el reactor se agrega agua $(50 \mathrm{~L})$ y se eleva la temperatura a $90^{\circ} \mathrm{C}$, se inicia la agitación para añadir ácido cítrico [0.9M], tiosulfato de sodio [0.5M] y se ajusta el $\mathrm{pH}$ a 3 con hidróxido de sodio. A diferencia del proceso anterior, solo se agregan $2.5 \mathrm{~kg}$ de arcilla caolinítica, manteniéndose así por un tiempo de 240 minutos, seguido de esto, se filtra la mezcla, separando la arcilla tratada y el licor de lixiviación, el cual, se regresa al reactor para nuevamente aumentar la temperatura a $90^{\circ} \mathrm{C}$, comenzar la agitación y agregar nuevamente $2.5 \mathrm{~kg}$ de arcilla caolinítica, este procedimiento se realiza tres ocasiones más, lixiviando en total de $10 \mathrm{~kg}$ de arcilla caolinítica con la misma solución.

Con el objetivo de simplificar el cálculo de los costos, sólo se analizaron aquellos que están directamente relacionados con la lixiviación, sin considerar procesos anteriores (disminución de tamaño de partícula, cribado, etc.) y posteriores (secado del caolín, empaquetado, etc.). 


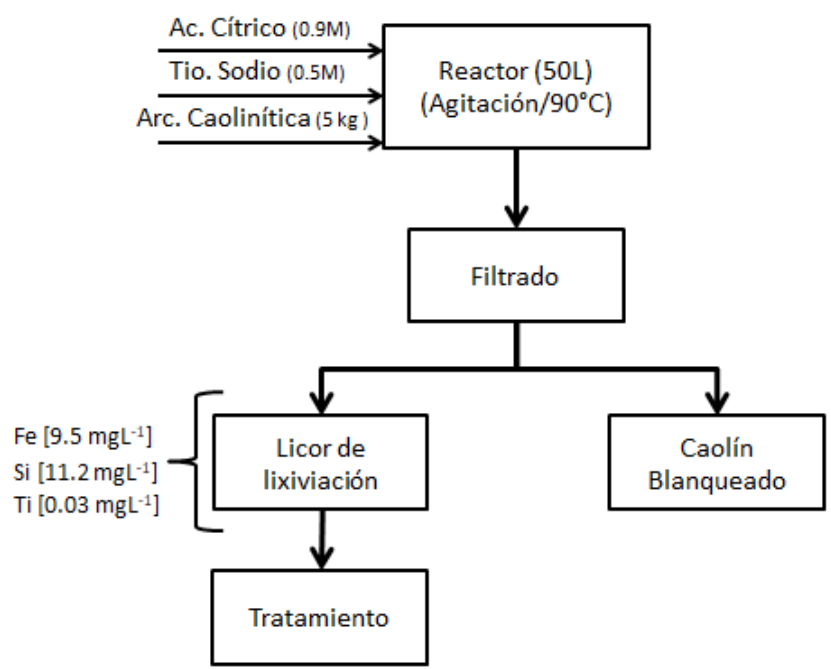

Figura 1: Diagrama del proceso 1 de la lixiviación de $5 \mathrm{~kg}$ de arcilla caolinítica.

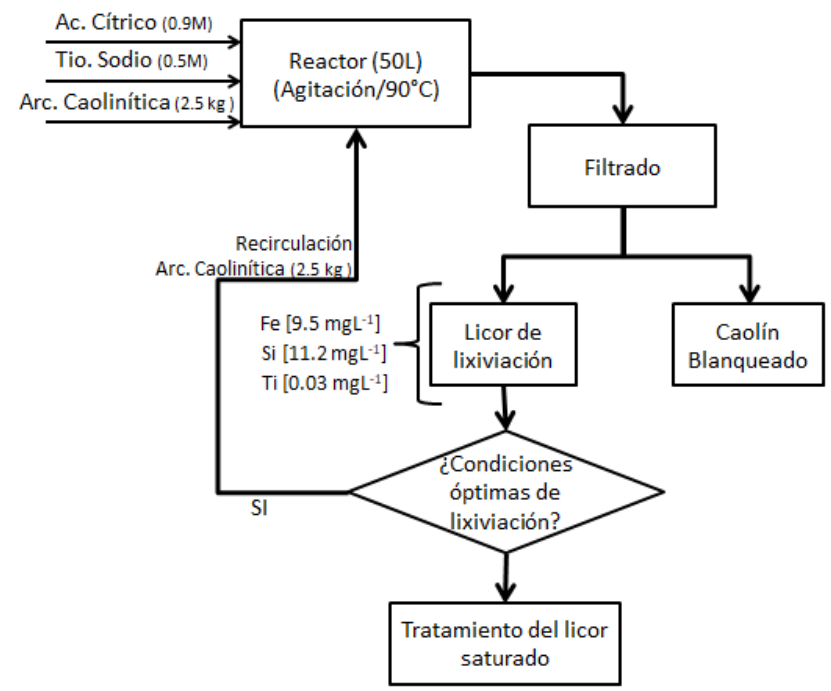

Figura 2: Diagrama del proceso 2 de la recirculación de los licores de lixiviación.

En la lixiviación se utilizó ácido cítrico de la marca sigmaaldrich, con $99 \%$ de pureza, el cual $100 \mathrm{~g}$ tienen un costo aproximado de $\$ 107.10$, el tiosulfato de sodio de la misma marca, con pureza de 99\%, $100 \mathrm{~g}$ tienen un costo de $\$ 161.00$ (precios revisados en febrero de 2018), la arcilla caolinítica (caolín con impurezas) a pie de mina tiene un precio entre los $\$ 200$ y $\$ 300$ por tonelada (Fondo Mixto CONACYT-Gobierno del Estado de Hidalgo, 2012) y cuando esta arcilla es de alta pureza, su precio asciende a $\$ 2,750,000$ la tonelada (SigmaAldrich, 2018). Es necesario mencionar que los costos de ácido cítrico y tiosulfato de sodio pueden disminuir al adquirir mayores cantidades de reactivos para el proceso de blanqueo a nivel industrial. De igual manera, es importante resaltar que la idea es automatizar la planta piloto de blanqueo de arcilla caolinítica (Sánchez, 2017), por lo que no se considerará el costo de la mano de obra para esta etapa en específico. Para el costo del agua se revisaron las cuotas del servicio industrial establecido por la Comisión Nacional del Agua (CONAGUA), específicamente en el estado de Hidalgo, que menciona un costo de $\$ 1051.04$ como cuota mínima de consumo de agua (de 0 a $20 \mathrm{~m}^{3}$ ). El costo de consumo de energía eléctrica $(\mathrm{kWh})$ se obtuvo de las tarifas establecidas por la Comisión Federal de Electricidad, para el tipo de tarifa "Gran demanda en media tensión horaria" (GDMTH) (CFE, 2018) y por la potencia eléctrica de $1.1 \mathrm{~kW}$ que utiliza un reactor de capacidad nominal de $50 \mathrm{~L}$ con revestimiento de vidrio, agitación y calentamiento (Quintaramon, 2018).

La cadena de valor de Porter visualizada en la Figura 3, solo se aplicará a la etapa de lixiviación de arcillas caoliníticas mediante una clasificación de aquellos factores que definen cada método, con el objetivo de determinar cuál de los dos propuestos aporta mayor beneficio o valor al producto o productos finales que se obtengan.

\begin{tabular}{|c|c|c|c|}
\hline & REACTIVOS & $\begin{array}{l}\text { PROCESODE } \\
\text { LIXIVIACIÓN }\end{array}$ & $\begin{array}{l}\text { PRODUCTO } \\
\text { FINAL }\end{array}$ \\
\hline & $\begin{array}{l}\text { - Ac. Cítrico }[0.9 \mathrm{M}] \\
\text { - Tio. Sodio }[0.5 \mathrm{M}] \\
\text { - Agua (50 L por } \\
\text { proceso) } \\
\text { - Arcilla caolinítica }\end{array}$ & $\begin{array}{l}\text { - Agitación } \\
\text { - Temperatura } 90^{\circ} \mathrm{C} \\
\text { - Tiempo }>90 \mathrm{~min} \text {. }\end{array}$ & $\begin{array}{l}\text { - Caolín blanqueado } \\
5 \mathrm{Kg} \\
\text { - } \text { Remoción de } \\
\text { contaminantes }<90 \%\end{array}$ \\
\hline 脑 & $\begin{array}{l}\text { - Ac. Cítrico [0.9M] } \\
\text { - Tio. Sodio [0.5M] } \\
\text { - Agua (50 L en } 4 \\
\text { procesos) } \\
\text { - Arcilla caolinítica }\end{array}$ & $\begin{array}{l}\text { - Agitación } \\
\text { - Temperatura } 90^{\circ} \mathrm{C} \\
\text { - Tiempo }=90 \mathrm{~min} . \\
\text { - } 3 \text { Recirculaciones del } \\
\text { licor de lixiviación }\end{array}$ & $\begin{array}{l}\text { - Caolín blanqueado } \\
10 \mathrm{Kg} \\
\text { - } \\
\text { Remoción de } \\
\text { contaminantes } \geq 99 \%\end{array}$ \\
\hline
\end{tabular}

Figura 3: Diagrama de la cadena de valor de los procesos de lixiviación.

\section{Resultados}

De acuerdo a la cadena de valor (Figura 3), es posible determinar que el proceso 2 basado en la recirculación de los licores de lixiviación, es el que agrega mayor valor al caolín blanqueado, pues se logra eliminar 99\% de contaminantes en un total de $10 \mathrm{~kg}$ de caolín. Además se utiliza una menor cantidad de agua en las lixiviaciones y la cantidad de reactivos a utilizar es mucho menor que al lixiviar $5 \mathrm{~kg}$ de arcilla caolinítica en un solo proceso.

Al hacer los cálculos tomando en consideración los precios y condiciones indicadas en la sección anterior, la evaluación se presenta de manera tabulada para cada uno de los procesos propuestos (Tabla 1 y Tabla 2); se desglosan los costos respectivos, considerando el precio de los reactivos, del agua, de la energía eléctrica y de la cantidad de arcilla caolinítica a lixiviar.

Al comparar las Tablas 1 y 2 , se muestra que el costo del proceso 2 (recirculación y lixiviación de $10 \mathrm{~kg}$ de arcilla caolinítica) es mayor, con un total de $\$ 15,714.21$ (MXN) en comparación a $\$ 15,648.67(\mathrm{MXN})$ que es el costo del proceso 1 (lixiviar $5 \mathrm{~kg}$ de arcilla caolinítica). La diferencia entre los costos, radica en el precio de la arcilla caolinítica a blanquear y por el consumo de energía eléctrica necesaria para cada lixiviación; es importante mencionar que la cantidad de ácido cítrico, tiosulfato de sodio y agua es la misma para ambos métodos. 
Tabla 1: Costos del proceso 1 para la lixiviación de las arcillas caoliníticas (el costo de cada insumo corresponde al utilizado en el experimento).

\begin{tabular}{lr}
\hline \multicolumn{1}{c}{ Insumos } & Precio $(\mathrm{MXN})$ \\
\hline Ácido Cítrico [0.9M] & $\$ 9,259.42$ \\
Tiosulfato de Sodio [0.5M] & $6,363.93$ \\
Agua (50L) & 2.65 \\
Energía Eléctrica & 21.43 \\
Arcilla Caolinítica $(5 \mathrm{~kg})$ & 1.25 \\
\hline Total $(5 \mathrm{~kg})=$ & $\$ 15,648.67$ \\
Total $(1 \mathrm{~kg})=$ & $\$ 3,129.73$ \\
\hline
\end{tabular}

Tabla 2: Costos del proceso 2 para la lixiviación de las arcillas caoliníticas (el costo de cada insumo corresponde al utilizado en el experimento).

\begin{tabular}{lr}
\hline \multicolumn{1}{c}{ Insumos } & Precio $(\mathrm{MXN})$ \\
\hline Ácido Cítrico [0.9M] & $\$ 9,259.42$ \\
Tiosulfato de Sodio $[0.5 \mathrm{M}]$ & $6,363.93$ \\
Agua (50L) & 2.65 \\
Energía Eléctrica & 85.71 \\
Arcilla Caolinítica $(10 \mathrm{~kg})$ & 2.50 \\
\hline Total $(10 \mathrm{~kg})=$ & $\$ 15,714.21$ \\
Total $(1 \mathrm{~kg})=$ & $\$ 1,571.42$ \\
\hline
\end{tabular}

Por último, cuando al precio de venta del caolín de alta pureza se le resta el valor del costo de cada proceso, se observa que es más beneficioso recircular los licores de lixiviación para saturar los mismo antes de darles un tratamiento adecuado y verterlos al medio ambiente. De este cálculo se obtiene una diferencia aproximada de $\$ 1,222.58$ (MXN) por kilogramo de arcilla caolinítica (Tabla 3).

Tabla 3. Cálculo de la diferencia del costo parcial y posible precio de venta por kilogramo al purificar la arcilla caolinítica, comparando el proceso 1 y el proceso 2 .

\begin{tabular}{lrr}
\hline \multicolumn{1}{c}{ Concepto } & $\begin{array}{c}\text { Proceso 1 } \\
\text { (Recirculación) }\end{array}$ & $\begin{array}{c}\text { Proceso 2 } \\
\text { (5 kg arcilla } \\
\text { caolinítica) }\end{array}$ \\
\hline Costo parcial & $\$ 1,571.42$ & $\$ 3,129.73$ \\
Precio de venta & $\$ 2,794.00$ & $\$ 1,397.00$ \\
\hline Diferencia $=$ & $\$ 1,222.58$ & $-\$ 1,732.73$ \\
\hline
\end{tabular}

\section{Conclusiones}

En este trabajo se efectuó la evaluación de costos en la etapa de lixiviación en un proceso de blanqueamiento de arcilla caolinítica con dos variantes, para lo cual se utilizaron los costos actualizados de los reactivos y recursos. Al comparar el costo total de cada proceso se observó que el proceso 2 representa un mayor costo por kilogramo de arcilla a tratar $(\$ 15,714.21 \mathrm{MXN})$, pero se logra el blanqueo de $10 \mathrm{~kg}$ de caolín en comparación con lixiviar $5 \mathrm{~kg}$ en una sola etapa $(\$ 15,648.67 \mathrm{MXN})$ (Proceso 1). Por último, de acuerdo al cálculo de la diferencia entre el posible precio de venta y los costos, se concluye que recircular los licores de lixiviación es un proceso que disminuye las afectaciones ambientales ya que se utiliza hasta 4 veces el licor para eliminar en cada proceso un $99 \%$ de contaminantes de la arcilla caolinítica, lo que genera un aumento al valor del producto final. Lo anterior da como resultado un mayor rango de ganancias que es el factor decisivo para determinar que el proceso 2 es el más rentable para escalar el proceso de blanqueamiento.

\section{English Summary}

\section{Cost evaluation in the leaching stage in a kaolinitic clay bleaching process.}

\begin{abstract}
In this work, a cost evaluation was carried out on the leaching stage of a kaolinitic clay bleaching process, for which two different methods of saturation of lixiviation liquors were analyzed. Both methods require constant stirring and a temperature of $90^{\circ} \mathrm{C}$ and dilute aqueous solutions of citric acid and sodium thiosulfate are used to remove the impurities present in the kaolinitic clay. In one of the procedures, $5 \mathrm{~kg}$ of kaolinitic clay were leached for periods greater than 240 minutes, which implies using more energy in the reactor; in a second procedure, the leach liquors were recirculated to bleach $10 \mathrm{~kg}$ of kaolinitic clay, without the need to add more reagents. Reagent costs, electric power consumption in the reactor, water consumption in industrial services and the cost of kaolinitic clay extracted at the mine pithead and of high purity were investigated. Comparing costs and analyzing the possible sale price, it was concluded that the most feasible method to bleach this clay is the recirculation of leach liquors to treat additional batches of clay.
\end{abstract}

Keywords:

Analysis, Costs, Kaolin, Leaching, Industrial plant

\section{Agradecimientos}

Este trabajo ha sido realizado parcialmente gracias al apoyo del Consejo Nacional de Ciencia y Tecnología (CONACyT) por la beca otorgada para realizar mis estudios de maestría. Agradezco a la Universidad Autónoma del Estado de Hidalgo (UAEH) por la apertura de programas enfocados en la investigación.

\section{Referencias}

Ambikandebi, V.R., Lalithambika, M., 2000. "Effect of organic acids on ferric iron removal from iron-stained kaolinite", Applied Clay Science, $16,3-4,133-145$

Arredondo, M. M. (2015). Contabilidad y Análisis de Costos (Ed. rev.). Cd. de México, México: Grupo Editorial Patria.

Cameselle, C., Nuñez, M. J., Lema, J. M., \& Pais, J. (1994). "Leaching of iron from kaolins by a spent fermentation liquor: influence of temperature, $\mathrm{pH}$, agitation and citric acid concentration". Journal of Industrial Microbiology, 14, 288-292.

Comisión Federal de Electricidad (CFE). 2018. "Tarifas industriales". Recuperado $2018, \quad$ marzo, de 
http://app.cfe.gob.mx/Aplicaciones/CCFE/Tarifas/Tarifas/tarifas_indus tria.asp

Departamento de Estadística Minera. (2010). Anuario Estadístico de la Minería Mexicana Ampliada 2009. Recuperado de https://www.gob.mx/cms/uploads/attachment/file/31734/anuario_2009. pdf, 208-209.

Frances, A. 2001. "Estrategias para la Empresa en América Latina". Caracas, Venezuela: Ediciones IESA. ISBN: 978-980-21-7244-3.

Gobierno del Estado de Hidalgo., 2017. "Periódico oficial, Comisión de Agua y Alcantarillado de Sistemas Intermunicipales". Recuperado 2 marzo, 2018, de http://201.116.60.81/Tarifas/Documentos/ Mineral\%20de\%20la\%20Reforma\%20Hgo\%202017.pdf

González J. A., Perino E., Ruiz M. del C., 2003. "Blanqueado de arcillas mediante la eliminación de hierro en la etapa de quemado", jornadas SAM, 09(10), 768-771.

Hernández, R. A., Legorreta, F., Hernández, L. E., Bedolla, A., 2015.'Kaolin Bleaching by Leaching Using Phosphoric Acid Solutions", Sociedad Química de México, 59(3), pp. 198-202.

Horngren, C. T., Datar, S. M., Foster, G., 2007. "Contabilidad de Costos. Un Enfoque Gerencial”. 12a Edición, México: Pearson- Prentice Hall. ISBN: 978-970-26-0761-8.

Jiménez, F. J., Espinoza C. L., 2007. "Costos Industriales". $1^{\text {a }}$ Edición, Costa Rica: Editorial Tecnológica de Costa Rica. ISBN: 9977-66-1839.

Legorreta, F., Salinas, E., Hernández L.E., Hernández R.A., Cerecedo E., 2015. "kinetics study of iron leaching from kaolinitic clay using oxalic acid", European Scientific Journal,11(12), 12-23.

Medinaceli, R., Trujillo, E., 2016. "Estudio técnico y económico de la explotación del yacimiento de Caolín perteneciente a la Carrera de Ingeniería de minas, petróleos y geotecnica de la U.T.O.”, Revista de Medio Ambiente Minero y Minería, Vol. 1, 46-55. ISSN: 2519-5352.

Olvera , P. N., Hernández , L. E., Lapidus , G. T., 2014. "Estudio del blanqueo de arcillas caoliníticas por medio de lixiviación”. Tesis Doctoral, Universidad Autónoma del Estado de Hidalgo, Área Académica de Ciencias de la Tierra y Materiales, Hidalgo, México

Olvera P. N., Hernández L. E., Lapidus G. T., 2012. "Estudio de la Remoción de Hierro de una Arcilla Caolinítica por medio de
Lixiviación Reductiva". XXI Congreso Internacional de Metalurgia Extractiva, 2-8.

Organización Mundial de la Salud. (2017, febrero). Chemical hazards in drinking-water. Recuperado 15 febrero, 2018, de http://www.who.int/water_sanitation_health/waterquality/guidelines/chemicals/en/\#H

Porter, M., 1986. "Ventaja Competitiva".2a Edición. México: Grupo Editorial Patria. ISBN: 0-02-925090-0

Quintaramon. (2018, 16 marzo). Cotizaciones. Recuperado 16 marzo, 2018, de http://www.quintaramon.com.mx/

Quintero, J., Sánchez J., 2006. "La cadena de valor: Una herramienta del pensamiento estratégico". TELOS. Revista de Estudios Interdisciplinarios en Ciencias Sociales. Vol. 8 (3), 77-389. ISSN: 1317-0570.

Sigma-Aldrich, 2018. "Citric Acid". Recuperado 26 febrero, 2018, de https://www.sigmaaldrich.com/catalog/product/sial/c0759?lang=es\&re gion=MX

Sigma-Aldrich, 2018. "Kaolin". Recuperado 26 febrero, 2018, de https://www.sigmaaldrich.com/catalog/product/sial/60609?lang=es\&re gion=MX

Sigma-Aldrich, 2018. "Sodium Thiosulfate". Recuperado 26 febrero, 2018, de https://www.sigmaaldrich.com/catalog/product/sigald/ 217263 ? lang=es\&region=MX

Subsecretaría de Minería, 2017. "Perfil de Mercado del Caolín”, Secretaría de Economía, 13-34.

Toache A. D., Hernández L. E., Lapidus G. T., Legorreta F., 2015. "Estudio de la posibilidad de Reutilización del Ácido Cítrico y Tiosulfato de Sodio utilizados en el Blanqueamiento de Arcillas Caoliníticas". XII Encuentro Participación de la Mujer en la Ciencia, 1-5. ISSN:2448-5063. 